\title{
A Kalman-Yakubovich-Popov-type lemma for systems with certain state-dependent constraints *
}

\author{
Christopher K. King ${ }^{\text {a }}$, Wynita M. Griggs ${ }^{\text {b }}$, Robert N. Shorten ${ }^{\text {b }}$ \\ ${ }^{a}$ Department of Mathematics, Northeastern University, Boston, MA 02115, USA \\ ${ }^{\mathrm{b}}$ Hamilton Institute, National University of Ireland, Maynooth, Co. Kildare, Ireland
}

\begin{abstract}
In this note, a result is presented that may be considered an extension of the classical Kalman-Yakubovich-Popov (KYP) lemma. Motivated by problems in the design of switched systems, we wish to infer the existence of a quadratic Lyapunov function (QLF) for a nonlinear system in the case where a matrix defining one system is a rank-1 perturbation of the other and where switching between the systems is orchestrated according to a conic partitioning of the state space $\mathbb{R}^{n}$. We show that a necessary and sufficient condition for the existence of a QLF reduces to checking a single constraint on a sum of transfer functions irrespective of problem dimension. Furthermore, we demonstrate that our conditions reduce to the classical KYP lemma when the conic partition of the state space is $\mathbb{R}^{n}$, with the transfer function condition reducing to the condition of Strict Positive Realness.
\end{abstract}

Key words: Kalman-Yakubovich-Popov lemma; nonlinear systems; switched systems; Lyapunov function; state space; state-dependent constraints; convex cone; frequency domain inequality; linear matrix inequality.

\section{Introduction}

The Kalman-Yakubovich-Popov (KYP) lemma has played a key role in many areas of systems theory over the past four decades $[1,5,10]$. The lemma establishes an equivalence between a frequency domain inequality (FDI) and a linear matrix inequality (LMI) which has proved to be useful in many areas of engineering and mathematics. Our objective in this paper is similar to that of [4]. In this paper, the authors impose constraints on the FDI and characterise these in terms of new LMIs. Motivated by problems in the design of switched systems however, we wish rather to impose constraints on the LMI and to investigate the consequences of these on the FDIs. In particular, we wish to solve the following problem. Suppose that $A$ is a Hurwitz matrix, with $b, c$ vectors. Suppose further that $\Omega$ is a region in $\mathbb{R}^{n}$ which is invariant under re-scaling $x \mapsto \lambda x$ for all $\lambda \neq 0$. We wish to obtain FDIs that can be used to determine

\footnotetext{
* This work was supported by SFI grant 07/IN.1/I901.

Email addresses: c.king@neu.edu (Christopher K. King), wynita.griggs@nuim.ie (Wynita M. Griggs), robert.shorten@nuim.ie (Robert N. Shorten).
}

whether there exists a $P=P^{T}>0$ such that

$$
\begin{aligned}
& \langle P x, A x\rangle<0 \text { for all } x \in \mathbb{R}^{n} \\
& \langle P x, A x\rangle<\langle P x, b\rangle\langle c, x\rangle \text { for all } x \in \Omega
\end{aligned}
$$

In doing this, we shall obtain necessary and sufficient conditions for the existence of such a $P$.

Before proceeding, it is worth noting that the Sprocedure [11] is a tool that has been used with much success to solve constrained Lyapunov equations [2,12]. Roughly speaking, using the S-procedure we reformulate the constrained Lyapunov function existence problem in a lossless manner, as a LMI, that can subsequently be solved using numerical techniques from convex optimization. While this approach is clearly of great merit, our results complement the use of the S-procedure. Recall that our approach is to replace the LMI altogether with a FDI and to infer the existence of a solution to the Lyapunov equation from this inequality. Not only does such an approach lead to insights that are extremely useful for control design but the existence of such a Lyapunov function is reduced to checking a single (albeit parameterised) FDI irrespective of problem dimension. 


\section{Preliminaries}

Our results borrow heavily from and extend our previous results on this topic. In particular, many similar ideas can be found in $[7,8]$. Our basic setup is as follows. Let $A$ be a Hurwitz matrix. Let $B$ be a Hurwitz matrix that is a rank-1 perturbation of $A$ :

$$
B=A-b c^{T}
$$

for some $b, c \in \mathbb{R}^{n}$, where $(A, b)$ is controllable and $(A, c)$ is observable. Ultimately, we wish to use a QLF to deduce the stability of the nonlinear system $\dot{x}=M(x) x$, $M(x) \in\{A, B\}$, where the switching between these matrices is orchestrated according to some partition of $\mathbb{R}^{n}$. In this context, let $\mathcal{C}$ be a convex polyhedral cone in $\mathbb{R}^{n}$, and let $\widehat{\mathcal{C}}$ denote its dual cone (or positive polar) defined by $\widehat{\mathcal{C}}=\left\{y: y^{T} x \geq 0\right.$ for all $\left.x \in \mathcal{C}\right\}$. It follows that $\widehat{\mathcal{C}}$ is also a convex polyhedral cone [3]. We will assume that the vector $c$ appearing in (3) belongs to the dual cone $\widehat{\mathcal{C}}$; that is, we assume

$$
B=A-b c^{T} \Rightarrow c^{T} x \geq 0 \text { for all } x \in \mathcal{C}
$$

The region $\Omega$ is defined as $\Omega=\mathcal{C} \cup(-\mathcal{C})$. In particular, we wish to determine the existence of a matrix $P=P^{T}>0$ such that $A^{T} P+P A<0$ and $x^{T}\left(B^{T} P+P B\right) x<0$ for all $x \in \Omega$.

Before we proceed to state the main result of the paper, we note the following lemmas that are useful in stating its proof. Our first result is an extension of the seminal result presented by Kamenetski and Pyanitski in [6]. It gives conditions under which certain types of convex cones intersect. This result is extremely useful in determining whether a QLF exists for certain types of stability problems.

Lemma 1 The necessary and sufficient condition that there exists a positive definite matrix $P$ satisfying (1) and (2) with $\Omega=\mathcal{C} \cup(-\mathcal{C})$ as defined above is that

$$
A W+W A^{T}+B y y^{T}+y y^{T} B^{T} \neq 0
$$

for every nonzero vector $y \in \Omega$ and $W=W^{T} \geq 0$.

The second result that we shall use provides a singularity test that can be used to determine the feasibility of the conditions given in the previous lemma. This result is an extension of our previous results derived in the context of the CQLF existence problem $[7,8]$.

Lemma 2 Let $y \in \mathbb{R}^{n}$ be a fixed vector. The following conditions are equivalent: (i) there exists a matrix $W=$ $W^{T} \geq 0$ satisfying

$$
A W+W A^{T}+B y y^{T}+y y^{T} B^{T}=0
$$

(ii) for some integer $r$, there are numbers $\alpha, \alpha_{1}, \ldots, \alpha_{r}$, $k_{1}, \ldots, k_{r}$ such that

$$
\left[B+\alpha^{2} A+\sum_{i=1}^{r} \alpha_{i}^{2}\left(A^{2}+k_{i}^{2} I\right)^{-1} A\right] y=0
$$

Equation (7) may be further simplified as follows. First, we substitute $B=A-b c^{T}$. This yields $\left[\left(1+\alpha^{2}\right) A+\right.$ $\left.\sum_{i=1}^{r} \alpha_{i}^{2}\left(A^{2}+k_{i}^{2} I\right)^{-1} A\right] y=\left(c^{T} y\right) b$. This implies

$$
\left[I+\sum_{i=1}^{r} \frac{\alpha_{i}^{2}}{1+\alpha^{2}}\left(A^{2}+k_{i}^{2} I\right)^{-1}\right] y=\frac{c^{T} y}{1+\alpha^{2}} A^{-1} b
$$

It is important to note that the operator on the left side of (8) can be inverted, and its inverse has a special form. This is summarized in the following lemma.

Lemma 3 Let $M$ be a real matrix with no eigenvalues in $(-\infty, 0]$. Let $0 \leq a_{1}<\cdots<a_{r}$ and $g_{1}, \ldots, g_{r}>0$. Then the matrix

$$
C=I+\sum_{i=1}^{r} g_{i}\left(M+a_{i} I\right)^{-1}
$$

is invertible, and

$$
C^{-1}=I-\sum_{i=1}^{r} h_{i}\left(M+b_{i} I\right)^{-1}
$$

where $h_{1}, \ldots, h_{r}>0, a_{1}<b_{1}<a_{2}<b_{2}<\cdots<b_{r}$, and

$$
\sum_{i=1}^{r} \frac{h_{i}}{b_{i}} \leq 1
$$

with equality in (11) if $a_{1}=0$. Conversely, given positive numbers $h_{1}, \ldots, h_{r}>0$ satisfying (11), the matrix on the right side of (10) is invertible, and its inverse has the form on the right side of (9) with positive numbers $g_{1}, \ldots, g_{r}$ and nonnegative numbers $a_{i}$ satisfying $0 \leq a_{1}<b_{1}<$ $a_{2}<b_{2}<\cdots<b_{r}$ with $a_{1}=0$ if equality holds in (11).

This previous lemma can be applied to (8) since $A$ is Hurwitz (and therefore $A^{2}$ has no real negative eigenvalues). Thus

$$
y=\frac{c^{T} y}{1+\alpha^{2}}\left[I-\sum_{i=1}^{r} h_{i}\left(A^{2}+\omega_{i}^{2} I\right)^{-1}\right] A^{-1} b
$$

It follows that the frequencies $\omega_{1}, \ldots, \omega_{r}$ and the coefficients $h_{1}, \ldots, h_{r}$ are all nonzero. Also, $\sum_{i=1}^{r} h_{i} / \omega_{i}^{2} \leq 1$ with equality if $k_{i}=0$ for any $i$. Thus, by defining 
$\gamma_{i}^{2}=\frac{h_{i}}{\omega_{i}^{2}}, i=1, \ldots, r$ and $\gamma_{0}^{2}=1-\sum_{i=1}^{r} \gamma_{i}^{2}$, and by introducing the frequency $\omega_{0}=0,(12)$ can be re-written as

$$
y=\frac{c^{T} y}{1+\alpha^{2}} \sum_{i=0}^{r} \gamma_{i}^{2}\left(A^{2}+\omega_{i}^{2} I\right)^{-1} A b
$$

Multiplying each side of (13) by $c^{T}$ implies $\sum_{i=0}^{r} \gamma_{i}^{2} c^{T}\left(A^{2}\right.$ $\left.+\omega_{i}^{2} I\right)^{-1} A b=1+\alpha^{2} \geq 1$. Define the sets

$$
\begin{aligned}
\mathcal{V}=\left\{\sum_{i=0}^{r} \gamma_{i}^{2}\left(A^{2}+\omega_{i}^{2} I\right)^{-1} A b: r \in \mathbb{N},\right. \\
\\
\left.\gamma_{0}, \ldots, \gamma_{r}, \omega_{0}, \ldots, \omega_{r} \in \mathbb{R}, \sum_{i=0}^{r} \gamma_{i}^{2}=1\right\}
\end{aligned}
$$

and

$$
\mathcal{S}(c)=\left\{v \in \mathcal{V}: c^{T} v \geq 1\right\}
$$

We summarize these observations in the following lemma.

Lemma 4 The necessary and sufficient condition that the vector $y$ satisfies (6) for some positive semidefinite matrix $W$ is that $y=\lambda v$ for some $\lambda \in \mathbb{R}$ and $v \in \mathcal{S}(c)$.

We will need one final preliminary result.

Lemma 5 The set $\mathcal{V}$ defined in (14) is convex and closed.

\section{Main Result}

We now state our main result. Recall that the FDI for the KYP lemma states that

$$
1+\operatorname{Re} c^{T}(j \omega I-A)^{-1} b>0
$$

for all real $\omega$ if and only if a certain LMI is feasible. Our main result extends this frequency domain condition to the case where this LMI in (1) and (2) is constrained in a certain manner. Recall that the dual cone $\widehat{\mathcal{C}}$ is also a convex polyhedral cone and thus there are vectors $h_{1}, \ldots, h_{N}$ whose positive linear span is $\widehat{\mathcal{C}}$. We will assume that $h_{1}, \ldots, h_{N}$ span $\mathbb{R}^{n}$, and thus $N \geq n$.

Theorem 1 Let $\Omega=\mathcal{C} \cup(-\mathcal{C})$, where $\mathcal{C}$ is a convex polyhedral cone satisfying (4). The following conditions are equivalent: (i) there exists a positive definite matrix $P$ satisfying $P A+A^{T} P<0$ and the constraint condition

$$
\langle P x, A x\rangle<\langle P x, b\rangle\langle c, x\rangle \text { for all nonzero } x \in \Omega
$$

(ii) there is a vector $v \in \widehat{\mathcal{C}}$ such that

$$
1+\operatorname{Re}(c+v)^{T}(j \omega I-A)^{-1} b>0
$$

for all $\omega \in \mathbb{R}$.
Comment 1 The main result says that there is a joint quadratic Lyapunov function (JQLF) for $\left(A, A-b c^{T}\right)$ in the region $\Omega$ if and only if there is a CQLF for the pair $\left(A, A-b(c+v)^{T}\right)$ where $v$ is some vector in $\widehat{\mathcal{C}}$. Namely:

(a) (JQLF) there exists a $P=P^{T}>0$ such that $A^{T} P+$ $P A<0$ and $x^{T}\left(\left(A-b c^{T}\right)^{T} P+P\left(A-b c^{T}\right)\right) x<0$ for all $x \in \Omega$;

(b) (CQLF) if and only if there exists a $P_{1}=P_{1}^{T}>0$ such that $A^{T} P_{1}+P_{1} A<0$ and $\left(\left(A-b w^{T}\right)^{T} P_{1}+P_{1}(A-\right.$ $\left.\left.b w^{T}\right)\right)<0$, where $w=c+v$ and $c, v$ are in $\widehat{\mathcal{C}}$.

Comment 2 If the positivity condition (4) is not satisfied in the cone $\mathcal{C}$ then it is still possible to find a constrained multi-dimensional frequency domain condition giving necessary and sufficient conditions for the existence of $P$, but the constraints become more complicated and onerous to check.

Comment 3 The main contribution of our result is to reduce the search for the existence of a JQLF to checking the existence of a CQLF for a pair of linear systems. This interpretation resembles many of the classical results in the literature. For example, the classical Popov criterion reduces the search for a Lur'e-Postnikov Lyapunov function to a positive realness condition on a parameter dependent transfer function, which in turn can be interpreted as a CQLF condition for a pair of linear systems [13].

Comment 4 The condition (18) is a generalization of the frequency condition of the KYP lemma. Indeed, suppose that $\Omega=\mathbb{R}^{n}$. Then $\Omega=\mathcal{C} \cup-\mathcal{C}$, where $\mathcal{C}=\{x$ : $\left.x^{T} c \geq 0\right\}$. In this case, $\widehat{\mathcal{C}}=\{\lambda c: \lambda \geq 0\}$, and so the condition $v \in \widehat{\mathcal{C}}$ in (ii) of the theorem is equivalent to $v=\lambda c$ for some $\lambda \geq 0$. Thus, the frequency condition (18) becomes $1+(1+\lambda) \operatorname{Re} c^{T}(j \omega I-A)^{-1} b>0$ for all $\omega \in \mathbb{R}$. This implies the usual KYP condition (16), and hence in this case the result of Theorem 1 reduces to the classical KYP Theorem.

Proof of Theorem 1: $(i) \Rightarrow(i i)$ : Suppose that there is a positive definite matrix $P$ satisfying (1) and (2). By Lemma 1, the left side of (5) must be nonzero for every nonzero vector $y \in \Omega$ and $W=W^{T} \geq 0$. Thus, by Lemma 2, the left side of (7) must be nonzero for every nonzero vector $y \in \Omega$, every integer $r$ and $\alpha, \alpha_{1}, \ldots, \alpha_{r}, k_{1}, \ldots, k_{r}$. By Lemma 4 , the left side of (7) is nonzero unless $y=\lambda v$ for some $\lambda \in \mathbb{R}$ and some $v \in \mathcal{S}(c)$. Thus $\mathcal{S}(c) \cap \Omega=\emptyset$.

Accordingly, suppose that a vector $v \in \mathcal{C}$ has the form

$$
v=\sum_{i=0}^{r} \gamma_{i}^{2}\left(A^{2}+\omega_{i}^{2} I\right)^{-1} A b=-\sum_{i=0}^{r} \gamma_{i}^{2} \operatorname{Re}\left(j \omega_{i} I-A\right)^{-1} b
$$


Then $v \notin \mathcal{S}(c)$, which means that $c^{T} v<1$. The condition $v \in \mathcal{C}$ can be written as $h_{a}^{T} v \geq 0$ for all $a=1, \ldots, N$ (recall that the vectors $h_{1}, \ldots, h_{N}$ generate the dual cone $\widehat{\mathcal{C}})$, or explicitly

$$
\sum_{i=0}^{r} \gamma_{i}^{2} \operatorname{Re} h_{a}^{T}\left(j \omega_{i} I-A\right)^{-1} b \leq 0 \text { for all } a=1, \ldots, N
$$

Thus, if (19) holds then also $c^{T} v<1$, or equivalently

$$
\sum_{i=0}^{r} \gamma_{i}^{2}\left[1+\operatorname{Re} c^{T}\left(j \omega_{i} I-A\right)^{-1} b\right]>0
$$

For every $\omega \in \mathbb{R}$, define the following vector in $\mathbb{R}^{N+1}$

$$
V(\omega)=\left(\begin{array}{c}
\operatorname{Re} h_{1}^{T}(j \omega I-A)^{-1} b \\
\vdots \\
\operatorname{Re} h_{N}^{T}(j \omega I-A)^{-1} b \\
1+\operatorname{Re} c^{T}(j \omega I-A)^{-1} b
\end{array}\right)
$$

and define the negative orthant in $\mathbb{R}^{N+1}: Q^{-}=\{x \in$ $\mathbb{R}^{N+1}: x_{i} \leq 0$ for all $\left.i=1, \ldots, N+1\right\}$. Then the conditions (20) and (21) above imply that

$$
\sum_{i=0}^{r} \gamma_{i}^{2} V\left(\omega_{i}\right) \notin Q^{-}
$$

for all $r$ and all $\left\{\gamma_{i}, \omega_{i}\right\}$. Define the following convex set in $\mathbb{R}^{N+1}: K=\left\{\sum_{i=0}^{r} \gamma_{i}^{2} V\left(\omega_{i}\right) \mid r \in \mathbb{N}, \gamma_{0}, \ldots, \gamma_{r}, \omega_{0}, \ldots\right.$, $\left.\omega_{r} \in \mathbb{R}, \sum_{i=0}^{r} \gamma_{i}^{2}=1\right\}$. Then (22) is equivalent to $K \cap$ $Q^{-}=\emptyset$. The set $Q^{-}$is a closed convex pointed cone, and $K$ is a compact convex set in $\mathbb{R}^{N+1}$ (closedness of $K$ follows from Lemma 5 and the observation that $K$ is the image of $\mathcal{V}$ under the map $v \mapsto\left(-h_{1}^{T} v, \ldots,-h_{N}^{T} v, 1-\right.$ $\left.c^{T} v\right)^{T}$, which is invertible by the assumption that the vectors $\left\{h_{a}\right\}$ span $\mathbb{R}^{n}$ ). Thus the condition that they do not intersect is equivalent to the condition that there is a hyperplane through the origin which strongly separates the sets $K$ and $Q^{-}$. Let $z$ be the normal vector of this separating hyperplane, then $z^{T} q \leq 0$ for all $q \in Q^{-}$, and $z^{T} y>0$ for all $y \in K$. The first condition implies that $z_{i} \geq 0$ for all $i$. Since every nonzero vector $y$ in $K$ is a positive linear combination of vectors $V\left(\omega_{i}\right)$, the second condition is equivalent to $z^{T} V(\omega)>0$ for all $\omega \in \mathbb{R}$. Equivalently, for all $\omega$

$$
\begin{aligned}
& z_{0}\left(1+\operatorname{Re} c^{T}(j \omega I-A)^{-1} b\right)+ \\
& \sum_{a=1}^{N} z_{a}\left(\operatorname{Re} h_{a}^{T}(j \omega I-A)^{-1} b\right)>0
\end{aligned}
$$

If $z_{0}>0$, then dividing by $z_{0}$ and defining $v=$ $\sum_{a=1}^{N}\left(z_{a} / z_{0}\right) h_{a}$ gives the result (18). If $z_{0}=0$, then note that there is $\omega_{\max }$ such that $1+\operatorname{Re} c^{T}(j \omega I-A)^{-1} b>0$ for all $|\omega| \geq \omega_{\max }$, and there is $\epsilon>0$ such that

$$
\begin{aligned}
& \epsilon\left(1+\operatorname{Re} c^{T}(j \omega I-A)^{-1} b\right)+ \\
& \sum_{a=1}^{N} z_{a}\left(\operatorname{Re} h_{a}^{T}(j \omega I-A)^{-1} b\right)>0
\end{aligned}
$$

for all $|\omega|<\omega_{\max }$. Thus in fact (24) holds for all $\omega$, and this implies (18) with $v=\sum_{a=1}^{N}\left(z_{a} / \epsilon\right) h_{a}$.

$($ ii $) \Rightarrow(i)$ : Now suppose there is a vector $v \in \widehat{\mathcal{C}}$ such that (18) holds for every $\omega$. Then there is a CQLF for the pair $\left(A, A-b(c+v)^{T}\right)$; hence for all $x \in \mathbb{R}^{n}$

$$
\langle P x, A x\rangle<0,\langle P x, A x\rangle<\langle P x, b\rangle(\langle c, x\rangle+\langle v, x\rangle)
$$

Let $x \in \mathcal{C}$, then $\langle c, x\rangle \geq 0$ and $\langle v, x\rangle \geq 0$. If $\langle P x, b\rangle \geq$ 0 , then $\langle P x, A x\rangle<0 \leq\langle P x, b\rangle\langle c, x\rangle$. If $\langle P x, b\rangle<0$, then from $(25),\langle P x, A \bar{x}\rangle<\langle P x, b\rangle(\langle c, x\rangle+\langle v, x\rangle) \leq$ $\langle P x, b\rangle\langle c, x\rangle$. Hence it is always true that $\langle P x, A x\rangle<$ $\langle P x, b\rangle\langle c, x\rangle$ for all $x \in \mathcal{C}$, and thus (i) holds for all $x \in \Omega$.

\section{Conclusions}

In this paper, we develop a KYP-like lemma. We constrain the classical KYP LMI and investigate the implication of this constraint on the FDI. Our results can be used to analyse state-dependent switching systems, including the case where the switching system is subject to parametric uncertainty [9].

\section{References}

[1] S. Boyd, L. El Ghaoui, E. Feron, and V. Balakrishnan. Linear Matrix Inequalities in System and Control Theory. SIAM, Philadelphia, PA, 1994.

[2] E. Feron. Quadratic stabilizability of switched systems via state and output feedback. Technical Report CIS-P-468, Center for Intelligent Control Systems, Brown University, Harvard University and MIT, 1996.

[3] M. Gerstenhaber. Activity Analysis of Production and Allocation, chapter XVIII. John Wiley \& Sons, New York, 1951.

[4] T. Iwasaki and S. Hara. Generalized KYP lemma: unified frequency domain inequalities with design applications. IEEE Transactions on Automatic Control, 50(1):41-59, 2005.

[5] R.E. Kalman. Lyapunov functions for the problem of Lur'e in automatic control. Proceedings of the National Academy of Sciences of the United States of America, 49(2):201-205, 1963.

[6] V.A. Kamenetskiy and Y.S. Pyatnitskiy. An iterative method of Lyapunov function construction for differential inclusions. Systems \& Control Letters, 8(5):445-451, 1987. 
[7] C. King and M. Nathanson. On the existence of a common quadratic Lyapunov function for a rank one difference. Linear Algebra and its Applications, 419(2-3):400-416, 2006.

[8] C. King and R. Shorten. Singularity conditions for the nonexistence of a common quadratic Lyapunov function for pairs of third order linear time invariant dynamic systems. Linear Algebra and its Applications, 413(1):24-35, 2006.

[9] C.K. King, W.M. Griggs, and R.N. Shorten. A result on the existence of quadratic lyapunov functions for state-dependent switched systems with uncertainty. In Proceedings of the 49th IEEE Conference on Decision and Control, pages 7339-7344, Atlanta, Georgia, USA, December 2010.

[10] S. Lefschetz. Stability of Nonlinear Control Systems. Academic Press, New York, NY, 1965.

[11] I. Pólik and T. Terlaky. A survey of the S-lemma. SIAM Review, 49(3):371-418, 2007.

[12] A. Rantzer and M. Johansson. Piecewise linear quadratic optimal control. IEEE Transactions on Automatic Control, 45(4):629-637, 2000.

[13] R.N. Shorten, P.F. Curran, and K. Wulff. On time-domain multiplier criteria for single-input single-output systems. International Journal of Control, 77(11):985-991, 2004.

\section{A Proof of Lemma 1}

The conditions (1) and (2) are

$$
\begin{aligned}
&\langle P x, A x\rangle<0 \text { all } x \neq 0 \in \mathbb{R}^{n}, \\
&\langle P y, B y\rangle<0 \text { all } y \neq 0 \in \Omega
\end{aligned}
$$

Necessity: Suppose that (A.1) holds for some $P=$ $P^{T}>0$ and let $y \neq 0 \in \Omega$ and $W=W^{T} \geq 0$. Write $W=$ $\sum_{i=1}^{n} w_{i} w_{i}^{T}$. Then (A.1) implies $\sum_{i=1}^{n}\left\langle P w_{i}, A w_{i}\right\rangle+$ $\langle P y, B y\rangle<0$. Use the cyclic property of the trace to deduce $\operatorname{Tr} P\left(A W+W A^{T}\right)+\operatorname{Tr} P\left(B y y^{T}+y y^{T} B^{T}\right)<0$ and hence $A W+W A^{T}+B y y^{T}+y y^{T} B^{T} \neq 0$.

Sufficiency: Suppose that (A.1) does not hold for any positive definite $P$. The Kamenetski-Pyanitski dual formulation [6] states that in this case there must exist nonzero vectors $x_{1}, \ldots, x_{k} \in \mathbb{R}^{n}$ and $y_{1}, \ldots, y_{m} \in \Omega$ for some $k, m$ such that

$$
A X+X A^{T}+B Y+Y B^{T}=0
$$

where $X=\sum_{i=1}^{k} x_{i} x_{i}^{T}$ and $Y=\sum_{i=1}^{m} y_{i} y_{i}^{T}$. Substituting $B=A-b c^{T}$ into (A.2) gives $A(X+Y)+(X+$ $Y) A^{T}-b(Y c)^{T}-(Y c) b^{T}=0$. Since $X+Y \neq 0$ by assumption, and $A$ is Hurwitz, it must hold that $Y c \neq 0$ and hence $\langle c, Y c\rangle>0$. Define $y=\langle c, Y c\rangle^{-1 / 2} Y c$ and $W=X+Y-y y^{T}$. Then $Y c=y y^{T} c$ and $y y^{T} \leq Y$. Hence $W \geq 0$ and $A W+W A^{T}+B y y^{T}+y y^{T} B^{T}=0$. Furthermore, $Y c=\sum_{i=1}^{m} y_{i} y_{i}^{T} c$. Without loss of generality the vectors $y_{i}$ may be chosen to belong to $\mathcal{C}$. By assumption (4) it follows that $y_{i}^{T} c \geq 0$. Since $\mathcal{C}$ is a convex cone it follows that $Y c \in \mathcal{C}$ and hence $y \in \mathcal{C} \subset \Omega$.

\section{B Proof of Lemma 2}

(i) $\Rightarrow$ (ii): Suppose that (6) holds for some $W=W^{T} \geq$ 0 . There is $\alpha \in \mathbb{R}$ such that $W \geq \alpha^{2} y y^{T}$ and $\operatorname{rank}(W-$ $\left.\alpha^{2} y y^{T}\right)=\operatorname{rank}(W)-1$. Let $Z=W-\alpha^{2} y y^{T}$. Then

$$
A Z+Z A^{T}+\left(B+\alpha^{2} A\right) y y^{T}+y y^{T}\left(B+\alpha^{2} A\right)^{T}=0
$$

The matrix $Z$ is singular and so $Z=\sum_{i=1}^{q} z_{i} z_{i}^{T}$ for some $q \leq n-1$, with independent vectors $z_{1}, \ldots, z_{q} \in \mathbb{R}^{n}$. By construction the vectors $y, z_{1}, \ldots, z_{q}$ are linearly independent. Using the results of Lemma 4 in [8] it follows that there are real numbers $\left\{s_{j k}, t_{j}\right\}(j, k=1, \ldots, q)$ with $s_{j k}=-s_{k j}$ such that

$$
\begin{aligned}
A z_{j}+\sum_{k \neq j} s_{j k} z_{k}=t_{j} y \quad(j=1, \ldots, q), \\
\left(B+\alpha^{2} A\right) y=-\sum_{j} t_{j} z_{j} .
\end{aligned}
$$

Let $S$ be the skew-symmetric matrix with entries $s_{i j}$. By an orthogonal transformation $Q$ it is possible to bring $S$ into the following block diagonal form

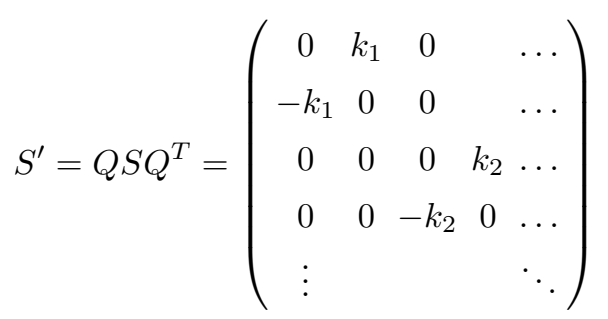

with at most $m=q / 2(\leq(n-1) / 2)$ nonzero $2 \times 2$ blocks on the diagonal, and all other entries zero. This follows from [8]. Letting $z_{i}^{\prime}=\sum_{j} Q_{i j} z_{j}$ and $t_{i}^{\prime}=\sum_{j} Q_{i j} t_{j}$ the equations (B.2) become

$$
\begin{aligned}
A z_{j}^{\prime}+\sum_{k \neq j} s_{j k}^{\prime} z_{k}^{\prime}=t_{j}^{\prime} y \quad(j=1, \ldots, q), \\
\left(B+\alpha^{2} A\right) y=-\sum_{j} t_{j}^{\prime} z_{j}^{\prime}
\end{aligned}
$$

We drop the primes henceforth. Solving (B.3) for $z_{1}, z_{2}$ gives

$$
\left(\begin{array}{l}
z_{1} \\
z_{2}
\end{array}\right)=\left(\begin{array}{cc}
A & k_{1} I \\
-k_{1} I & A
\end{array}\right)^{-1}\left(\begin{array}{l}
t_{1} y \\
t_{2} y
\end{array}\right)
$$

and similarly for the successive pairs $z_{3}, z_{4}, \ldots, z_{2 m-1}$, $z_{2 m}$. For the remaining variables the solution is $z_{i}=t_{i} A^{-1} y$. Substituting these into the equation for $y$ gives $\left(B+\alpha^{2} A\right) y=-\sum_{i=1}^{m}\left(\begin{array}{ll}t_{2 i-1} & t_{2 i}\end{array}\right)\left(\begin{array}{c}z_{2 i-1} \\ z_{2 i}\end{array}\right)-$ $\sum_{j=2 m+1}^{q} t_{j}^{2} A^{-1} y=-\alpha_{0}^{2} A^{-1} y-\sum_{i=1}^{m} \alpha_{i}^{2}\left(A^{2}+\right.$ 
$\left.k_{i}^{2} I\right)^{-1} A y=-\sum_{i=0}^{m} \alpha_{i}^{2}\left(A^{2}+k_{i}^{2} I\right)^{-1} A y$ where $\alpha_{i}^{2}=$ $t_{2 i-1}^{2}+t_{2 i}^{2}$ for $i=1, \ldots, m, \alpha_{0}^{2}=\sum_{j=2 m+1}^{q} t_{j}^{2}$ and $k_{0}=0$. After relabeling summation indices this gives (7).

$($ ii $) \Rightarrow(i)$ : Suppose that $(7)$ holds. Define $Z=$ $\sum_{i=1}^{r} \alpha_{i}^{2}\left(A^{2}+k_{i}^{2} I\right)^{-1}\left[A y y^{T} A^{T}+k_{i}^{2} y y^{T}\right]\left(\left(A^{2}+k_{i}^{2} I\right)^{-1}\right)^{T}$. Then $Z \geq 0$. Multiplying $Z$ on the left hand side by $A$, then alternatively on the right hand side by $A^{T}$, and then adding the two terms $A Z$ and $Z A^{T}$ gives

$$
\begin{aligned}
A Z+Z A^{T}=\sum_{i=1}^{r} & \alpha_{i}^{2}\left[\left(A^{2}+k_{i}^{2} I\right)^{-1} A y y^{T}\right. \\
& \left.+y y^{T} A^{T}\left(\left(A^{2}+k_{i}^{2} I\right)^{-1}\right)^{T}\right]
\end{aligned}
$$

Furthermore from (7) it follows that

$$
\begin{aligned}
(B & \left.+\alpha^{2} A\right) y y^{T}+y y^{T}\left(B+\alpha^{2} A\right)^{T}=-\sum_{i=1}^{r} \alpha_{i}^{2}\left[\left(A^{2}\right.\right. \\
& \left.\left.+k_{i}^{2} I\right)^{-1} A y y^{T}+y y^{T} A^{T}\left(\left(A^{2}+k_{i}^{2} I\right)^{-1}\right)^{T}\right]
\end{aligned}
$$

Combining (B.4) and (B.5) gives (B.1), which implies (6) with $W=Z+\alpha^{2} y y^{T}$.

\section{Proof of Lemma 3}

Define the function

$$
f(x)=1+\sum_{i=1}^{r} g_{i}\left(x+a_{i}\right)^{-1}
$$

By sketching the graph of $f$ and noting that $g_{i}>0$ for all $i$, it follows that $f$ has $r$ real zeroes $-b_{1}, \ldots,-b_{r}$ satisfying $a_{1}<b_{1}<a_{2}<b_{2}<\cdots<b_{r}$. Thus $f$ can be written as the ratio of polynomials $f(x)=\prod_{i=1}^{r} \frac{x+b_{i}}{x+a_{i}}$ and hence $f(x)^{-1}=\prod_{i=1}^{r} \frac{x+a_{i}}{x+b_{i}}$. We now claim that

$$
f(x)^{-1}=1-\sum_{i=1}^{r} h_{i}\left(x+b_{i}\right)^{-1}
$$

where $h_{i}>0$ for all $i$ and

$$
\sum_{i=1}^{r} \frac{h_{i}}{b_{i}} \leq 1
$$

The first claim (C.2) is proven by induction on $r$. For $r=1$ we have $\frac{x+a_{1}}{x+b_{1}}=1-\frac{b_{1}-a_{1}}{x+b_{1}}=1-\frac{h_{1}}{x+b_{1}}$ where $h_{1}=b_{1}-a_{1}>0$, hence the result is shown for this case. For the induction step assume that (C.2) holds for all $r \leq n$. Then $\prod_{i=1}^{n+1} \frac{x+a_{i}}{x+b_{i}}=\prod_{i=1}^{n} \frac{x+a_{i}}{x+b_{i}} \frac{x+a_{n+1}}{x+b_{n+1}}=$ $\left(1-\sum_{i=1}^{n} \frac{h_{i}}{x+b_{i}}\right) \frac{x+a_{n+1}}{x+b_{n+1}}=1-\sum_{i=1}^{n} \frac{h_{i}^{\prime}}{x+b_{i}}-\frac{h_{n+1}^{\prime}}{x+b_{n+1}}$, where $h_{i}^{\prime}=g_{i} \frac{a_{n+1}-b_{i}}{b_{n+1}-b_{i}}$ for $i=1, \ldots, n$ and $h_{n+1}^{\prime}=\left(b_{n+1}-\right.$ $\left.a_{n+1}\right)\left[1+\sum_{j=1}^{n} \frac{g_{j}}{b_{n+1}-b_{j}}\right]$. It follows that $h_{i}^{\prime}>0$ for $i=1, \ldots, n+1$ and this completes the induction step. Finally, (C.1) implies that $f(0) \geq 1$ and hence $0 \leq$ $f(0)^{-1} \leq 1$, which is (C.3).

Conversely, starting with $1-\sum_{i=1}^{r} h_{i}\left(x+b_{i}\right)^{-1}$ satisfying (C.3), a sketch of the graph shows that the function has $r$ real zeroes at positions $-a_{1}, \ldots,-a_{r}$ satisfying $0 \leq a_{1}<b_{1}<a_{2}<b_{2}<\cdots<b_{r}$ with $a_{1}=0$ if and only if $\sum_{i=1}^{r} h_{i} / b_{i}=1$. Hence it can be written $1-\sum_{i=1}^{r} h_{i}\left(x+b_{i}\right)^{-1}=\prod_{i=1}^{r} \frac{x+a_{i}}{x+b_{i}}$. Then an argument similar to the previous shows that the inverse has the form on the right side of (C.1).

This proves Lemma 3 for the case where $M=x$ is a positive number. To see that the general case follows, note that the result of Lemma 3 can be stated equivalently as

$$
\begin{gathered}
\left(I+\sum_{i=1}^{r} g_{i}\left(M+a_{i} I\right)^{-1}\right)\left(I-\sum_{i=1}^{r} h_{i}\left(M+b_{i} I\right)^{-1}\right. \\
-I=0
\end{gathered}
$$

By multiplying across by $\prod_{i=1}^{r}\left(M+a_{i} I\right)\left(M+b_{i} I\right)$ the terms with inverses can be removed. After multiplying out the expressions what remains are sums of powers of $M$, and thus the left side of (C.4) becomes a polynomial $P(M)$. Since the result is known to hold for $M=x$, we have $P(x)=0$ for all $x \geq 0$. Hence $P=0$, and thus (C.4) holds.

\section{Proof of Lemma 5}

Recall the definitions (14) and (15). Clearly $\mathcal{V}$ is convex. Furthermore, suppose $v$ is in the closure of $\mathcal{V}$. Then there is a Cauchy sequence $v_{k} \in \mathcal{V}$ and a vector $d \in \mathbb{R}^{n}$ such that $v_{k} \rightarrow v$ and such that $d^{T} v \geq 1, d^{T} v_{k} \geq 1$ for all $k$. Thus $v_{k} \subset \mathcal{S}(d)$, and so by Lemma 4 , for each $k$ there is $W_{k}=W_{k}^{T} \geq 0$ such that equation (6) holds, with $B=A-b d^{T}$. The relation (6) can be written as $W_{k}=-L_{A}^{-1} L_{B}\left(v_{k} v_{k}^{T}\right)$ where $L_{A}(\cdot)=A(\cdot)+(\cdot) A^{T}$, and similarly for $L_{B}$. Thus $\left\{W_{k}\right\}$ is a Cauchy sequence in the set of positive semidefinite matrices and therefore (by closedness) converges to some $W \geq 0$. By continuity $W=-L_{A}^{-1} L_{B}\left(v v^{T}\right)$, and thus $v \in \mathcal{S}(d) \subset \mathcal{V}$. Therefore $\mathcal{V}$ is closed. 